\title{
Photosensitivität bei Pellagroid unter tuberkolostatischer Therapie mit Pyrazinamid
}

\author{
Photosensitivity with Pellagroid During Tubercolosis Chemotherapy \\ with Pyrazinamide
}

Autoren

Institut

\section{Kowalzick, L. Eickenscheidt, A. Büttner, B. Schell}

Klinik für Hautkrankheiten und Allergologie (Chefarzt: Prof. Dr. med. habil. L. Kowalzick) HELIOS Vogtland-Klinikum Plauen GmbH (Ärztlicher Direktor: Prof. Dr. med. habil. L. Kowalzick)

\section{Bibliografie}

DOI $10.1055 / \mathrm{s}-0028-1103431$

Online-Publikation: 13.1.2009

Akt Dermatol 2009; 35:

180-183 @ Georg Thieme

Verlag KG Stuttgart · New York ISSN 0340-2541

Korrespondenzadresse

Prof. Dr. med. habil.

Lutz Kowalzick

Klinik für Hautkrankheiten

und Allergologie

HELIOS Vogtland-Klinikum

Plauen GmbH

Postfach 100153

08505 Plauen

lutz.kowalzick@

helios-kliniken.de

\section{Zusammenfassung}

$\nabla$

Wir berichten über einen 73-jährigen Patienten, der unter einer tuberkolostatischen Kombinationstherapie zunächst ein makulopapulöses Arzneimittelexanthem und danach ein Pellagroid in lichtexponierten Hautarealen entwickelte. Ur-

\section{Einleitung}

Die Tuberkulose erlebte im letzten Jahrzehnt eine Renaissance mit einer Zunahme um ca. $20 \%$ binnen 5 Jahren [4]. Ursächlich hierfür könnten die Verringerung des BCG-geimpften Anteils der Bevölkerung, die Erhöhung des Anteils von Migranten und sozial benachteiligten Personen, die HIVInfektion sowie die weitere Verbreitung von immunsuppressiv wirkenden Medikamenten sein. Deswegen werden auch wieder häufiger Antituberkulosemittel therapeutisch eingesetzt. Dermatologen sollten daher die möglichen Nebenwirkungen dieser Präparate auf das Hautorgan bewusst sein, und durch deren Kenntnis in Kooperation mit den behandelnden Pulmonologen frühzeitig intervenieren, auch um schweren systemischen Symptomen wie bei der Pellagra vorzubeugen.

\section{Kasuistik \\ $\nabla$}

Wir berichten über einen 73-jährigen Patienten, bei dem seit vier Monaten eine vierfache Tuberkulostatika-Kombinationstherapie mit Ethambutol $1200 \mathrm{mg}$, Pyrazinamid $2500 \mathrm{mg}$, Isoniazid $300 \mathrm{mg}$ und Rifampicin $600 \mathrm{mg}$ tgl. wegen einer nicht offenen, histologisch gesicherten Lungentuberkulose durchgeführt wurde. Zwei Wochen später entwickelte sich ein stammbetontes deutlich juckendes makulopapulöses Exanthem, woraufhin nach etwa sechs Wochen zunächst sächlich für letzteres dürfte die Einnahme von Pyrazinamid sein. Bereits sechs Tage nach Beginn eines konsequenten Lichtschutzes und drei Tage nach Absetzen des Präparates kam es auch ohne weitere spezifische Therapie zur weitgehenden Abheilung der Herde.

Ethambutol und Pyrazinamid (Pyrafat ${ }^{\circledR}$ ), die von den vorbehandelnden Kollegen als hierfür wahrscheinlich ursächlich angesehen wurden, abgesetzt wurden. Trotz dieser Maßnahme und lokaler Kortikosteroid-Anwendungen kam es zu einem weiteren deutlichen Progress des Exanthems. Daraufhin wurden nach sechs Wochen auch Isoniazid und Rifampicin abgesetzt und der Patient stationär eingewiesen.

Bei Aufnahme fand sich eine Suberythrodermie mit Hyperkeratosen und Rhagaden palmoplantar. Die peripheren Lymphknoten fanden sich palpatorisch nicht vergrößert.

Auch zum Ausschluss eines kutanen T-Zell-Lymphoms wurde eine Probebiopsie durchgeführt. Histologisch fanden sich Veränderungen (herdförmige Spongiose, perivaskuläres und interstitielles lymphohistiozytäres Infiltrat mit vereinzelten eosinophilen Granulozyten), die mit einem Arzneimittelexanthem vereinbar waren. Wir behandelten lokal initial mit Methylprednisolonaceponat-Creme im Gesicht und Triamcinolon 0,1 \% in Ungt. leniens am Körper sowie mit Argentum nitricum und Urea $10 \%$ in Unguentum leniens palmoplantar. Wegen der Grunderkrankung verzichteten wir auf die systemische Gabe von Kortikosteroiden. Symptomatisch gaben wir intern Clemastin. Nach Rücksprache mit den behandelnden Pulmonologen begannen wir unter stationärer Kontrolle mit der erneuten Gabe von Ethambutol und Pyrazinamid. Eine vorherige epikutane Testung war wegen der Suberythrodermie nicht möglich. Trotz der Reexposition 
mit diesen Antituberkulosepräparaten kam es, auch unter Ausschleichen der Lokaltherapie und schließlichem Umsetzen auf pflegende Externa allein, binnen 14 Tagen allmählich zu einer vollständigen Abheilung und zu keinem Rezidiv des Exanthems. Wir entließen den Patienten in ambulante Weiterbehandlung mit der Maßgabe, ihn etwa 4 Wochen nach dem Ende der lokalen Steroidanwendungen zur Epikutantestung und ggf. oralen Expositionstestung von Isoniazid und Rifampicin wiedervorzustellen, da die Pulmonologen zumindest mit einer tuberkulostatischen Dreifach-Kombination weiter behandeln wollten.

Eine Woche vor der beabsichtigten Testung wurde der Patient dann Anfang August während einer hochsommerlichen Schönwetterperiode mit unter Ethambutol und Pyrazinamid neu aufgetretenen Hautveränderungen vorgestellt. Er klagte über Juckreiz der Herde. Diarrhoen wurden nicht angegeben. Es fanden sich flächige, unscharf begrenzte und gering schuppende Erytheme an den Streckseiten der Hände ( $\bullet$ Abb. 1 a), am Capillitium, an den Schläfen, an der Stirn, dem Nasenrücken und den Unterlidern, am Dekolleté sowie an den Streckseiten der Unterschenkel und proximalen Fußrücken. Die Unterlippe fand sich deutlich geschwollen, gerötet und erosiv und war großenteils von einer grau-weißlichen bis hämorrhagisch tingierten Kruste bedeckt ( Abb. 2a). Das Nikotinamid im Serum fand sich drei Tage nach Beendigung der Pyrazinamideinnahme mit $9 \mathrm{ng} / \mathrm{ml}$ am untersten Rand des Normbereichs (8-52 ng/ml). Der Metabolit Vitamin B6 (Pyridoxol) fand sich im Sammelurin mit $0,27 \mu \mathrm{mol} / 24 \mathrm{~h}$. (Normbereich $\geq 5 \mu \mathrm{mol} / 24 \mathrm{~h}$ ) deutlich erniedrigt. Die 5-Indolessigsäure zum Ausschluss eines Karzinoids und die Gesamtporphyrine zum Ausschluss einer Porphyrie waren im Sammelurin negativ bzw. im Normbereich. Antinukleäre Faktoren und dsDNS-Antikörper fanden sich negativ. Die Erythemschwellen für UV-A und UV-B waren auch in der Ablesung nach 72 Stunden unauffällig.

Aufgrund der bekannten photosensibilisierenden Eigenschaften von Pyrazinamid und der Morphe und insbesondere Lokalisation der aktuellen Hautveränderungen stellten wir die Verdachtsdiagnose eines medikamentös induzierten Pellagroids und empfahlen dem Patienten Lichtschutz durch entsprechendes Meidungsverhalten auch hinter (Auto-) Fensterglas, geeignete lange Bekleidung und Kopfbedeckung sowie die topische Anwendung eines Sunblockers mit hoher Schutzwirkung gegen UV-B und UV-A (Anthelios XL $50^{+}$Crème Fondante ${ }^{\circledR}$ ). Drei Tage später wurde die Pyrazinamid-Medikation durch die Pulmonologen abgesetzt. Bei Wiedervorstellung drei Tage später fanden sich sämtliche Haut- und Schleimhautveränderungen ohne weitere spezifische Therapie weitestgehend abgeheilt ( $\bullet$ Abb. $\mathbf{1}$ b und $\mathbf{2}$ b). In der anschließenden Epikutantestung ließ sich keine Kontaktsensibilisierung vom Spättyp gegen Isoniazid und Rifampicin nachweisen. Auf eine orale Provokationstestung mit diesen Substanzen wurde zunächst verzichtet, da eine Fortsetzung der tuberkulostatischen Therapie seitens der Pulmonologen nicht mehr für erforderlich gehalten wurde, und somit die Gefahr einer Rezidivauslösung des Arzneimittelexanthems durch die Provokation unverhältnismäßig gewesen wäre.

\section{Diskussion \\ $\nabla$}

Als Pellagra wird eine polysymptomatische Erkrankung bezeichnet, die aufgrund eines Mangels an der essenziellen Aminosäure Tryptophan bzw. des Vitamins B3 (Nikotinsäureamid, Nikotinamid) aufgrund Malnutrition (z.B. durch einseitige Mais- oder
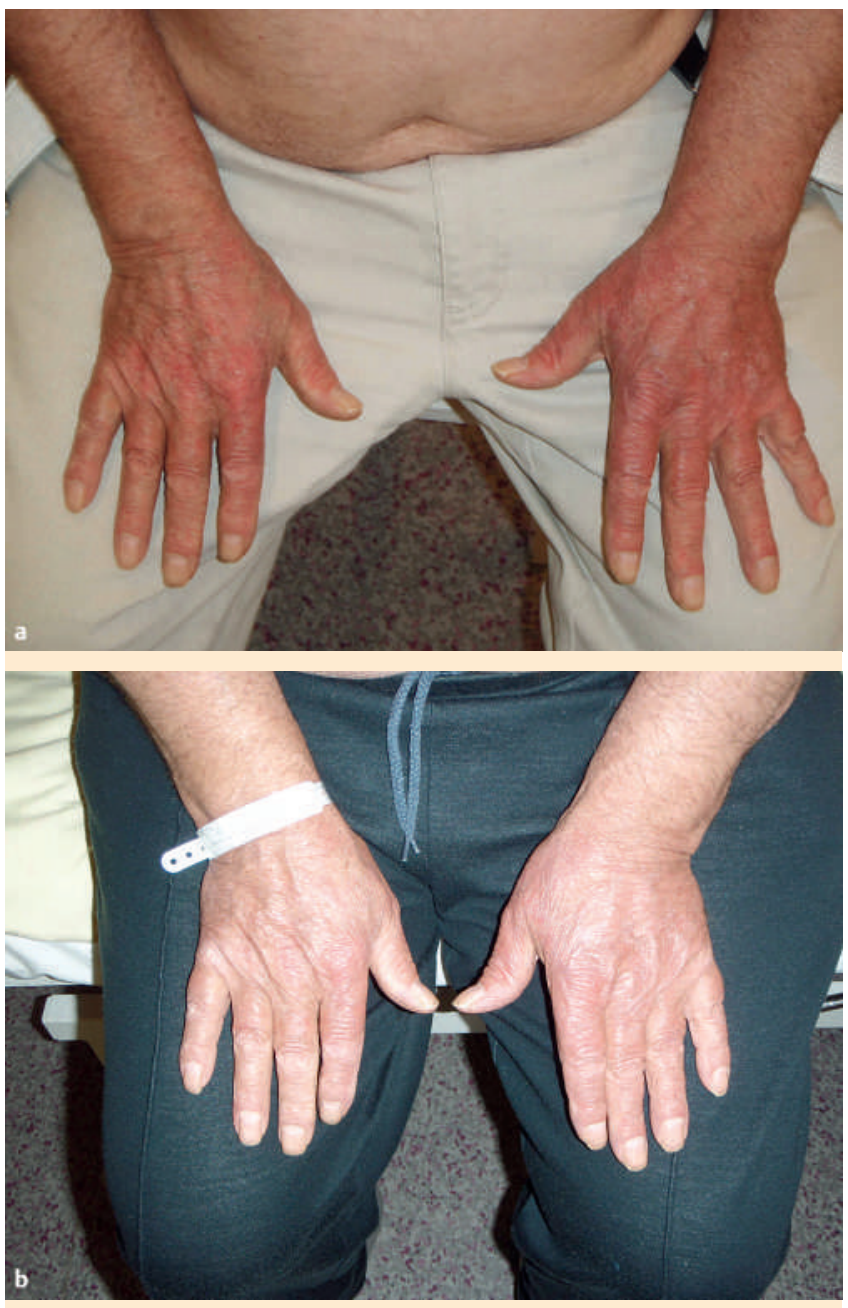

Abb. 1 Hochsommerlich lichtexponierte Handrücken eines 73-jährigen Patienten unter einer oralen tuberkulostatischen Therapie mit Pyrazinamid (a) und sieben Tage nach Beginn konsequenten Lichtschutzes bzw. drei Tage nach Absetzen des Medikaments (b). Pellagroide Erytheme mit Schuppung und rascher Rückbildung ohne weitere spezifische Therapie.

Reisernährung) oder einer Resorptionsstörung (Harnup-Syndrom) entsteht. Weitere, sekundäre Ursachen für Tryptophanbzw. Nikotinamidmangel sind das Karzinoid, chronisch-entzündliche Darmerkrankungen, chronische Diarrhoen, AIDS, chronischer Alkoholismus und verschiedene Medikamente. $\mathrm{Zu}$ letzteren gehören neben einigen Chemotherapeutika und Psychopharmaka auch die Tuberkulostatika Isoniazid, Pyrazinamid und Ethionamid. Letztere Substanzen sind vermutlich aufgrund ihrer chemisch ähnlichen Struktur kompetitiv zu Nikotinamid bei der Synthese von Nikotinamid-Adenosid-Dinukleotid-Phosphat (NADP), einem essenziellen Koenzym der zellulären Atmungskette, und bei der Synthese von Pyrimidinen und von Vitamin B6 (Pyridoxol). Die klinischen Symptome bei der Pellagra können sich in den drei „D“ Dermatitis, Diarrhoe und Demenz manifestieren, also in Organsystemen, in denen ein besonders hoher Zellumsatz oder ein besonders hoher Energiebedarf besteht.

In etwa $80 \%$ der Pellagra-Fälle sind Hautsymptome vorhanden, die sich typischerweise initial als Erytheme in lichtexponierten Hautarealen, wie dem Gesicht, dem Dekolleté und den Streckseiten von Unterarmen und Händen sowie von Unterschenkeln und Füßen, manifestieren. Die Erytheme können vesikulo-bullös, 


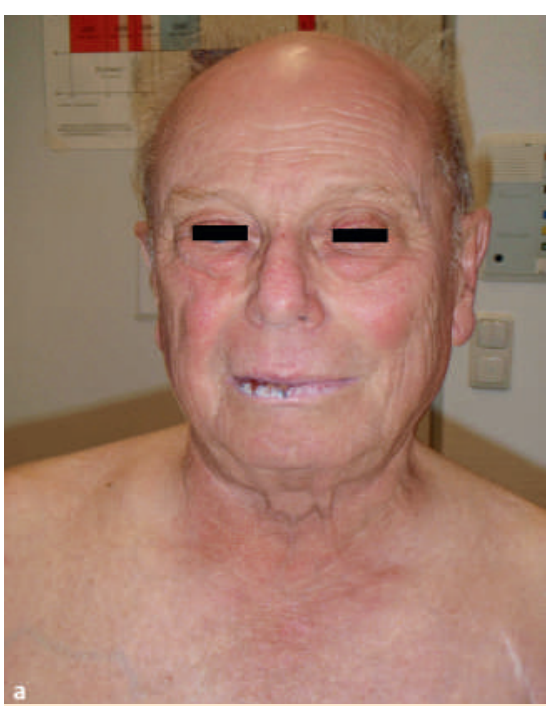

Abb. 2 Gesicht mit Unterlippe sowie Dekolleté desselben Patienten unter Therapie mit Pyrazinamid (a) und nach Beginn konsequenten Lichtschutzes bzw. Absetzen des Medikaments (b). Pellagroide Erytheme mit Schuppung periorbital und am Nasenrücken und am Dekolleté und ödematöser Lippenschwellung mit Krustenbildung und rascher Rückbildung ohne weitere spezifische Therapie.

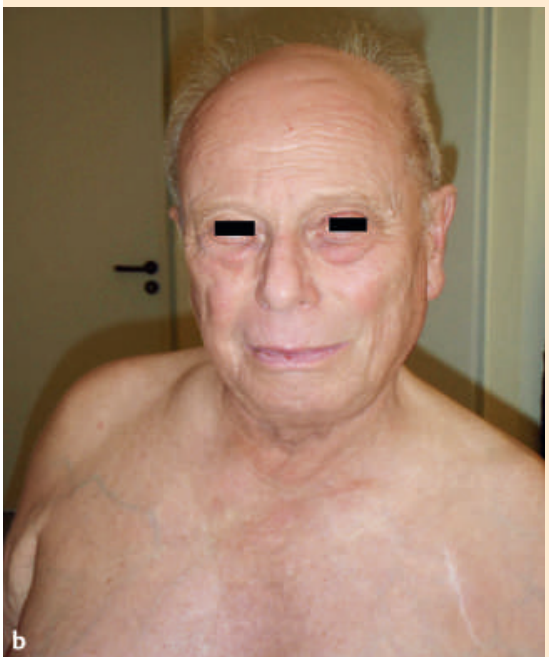

verkrustet und deutlich ödematös geschwollen sein. Im weiteren Verlauf über einige Wochen können Hyperpigmentierungen (Pellagra-Maske, Halsband von Casàl [Erstbeschreiber 1762 bzw. Stadt in Süditalien]), pergamentartige Atrophie und Trockenheit sowie eine starke Schuppung die Folge sein. Dieser typische $\mathrm{Zu}-$ stand des pellagroiden Erythems erlaubt häufig die Diagnose, bevor es zum Auftreten gastroenterologischer oder neuropsychiatrischer Symptome kommt. Auch Glossitis und Dysphagien können vorkommen. Unbehandelt kann die Pellagra in 4 bis 5 Jahren zum Tode durch Kachexie führen [9]. Zu den klinischen Differenzialdiagnosen des pellagroiden Erythems zählen der kutane Lupus erythematodes, die Porphyria cutanea tarda, andere Photodermatosen und Pemphiguserkrankungen [6].

Der genaue Mechanismus der Photosensibilisierung bedingt durch Vitamin B3-Mangel ist unbekannt. Vermutet kann jedoch werden, dass die Schädigung des Energiestoffwechsels und der Pyrimidin-Nukleotidsynthese die Reparaturmechanismen des Hautorgans auf UV-induzierte Schädigungen beeinträchtigen. Pyrazinamid ist ein tuberkulozid wirkendes Chemotherapeutikum, das in Mehrfachkombination neben anderen Tuberkulostatika oder als Reservemedikament bei anderwärtig resistenten Mycobacterium-tuberculosis-Stämmen eingesetzt wird, da keine Kreuzresistenzen zu den meisten gängigen Tuberkulostatika bekannt sind. Der genaue Wirkmechanismus ist unbekannt, es wird jedoch im Tuberkelbazillus intrazellulär aufgrund seiner Ähnlichkeit mit Nikotinamid enzymatisch in die ebenfalls antimykobakteriell wirksame Pyrazincarbonsäure umgewandelt. Unseres Wissens wurde erst ein Fall einer durch Pyrazinamid im Rahmen einer Polychemotherapie mit Antitubekulosemitteln induzierten Pellagra explizit publiziert [7]; laut Herstellerangaben wurde eine Photosensibilisierung mit Auftreten von Hauterscheinungen aber häufiger gemeldet. Demgegenüber sind Dutzende Fälle von Pellagra und pellagroide Erythmen unter dem chemisch ähnlichen Tuberkulostatikum Isoniazid in der Literatur beschrieben [10]. Möglicherweise bewirkt bei einem Teil dieser Patienten ein homozygoter Defekt der N-Acetyltransferase eine verminderte Metabolisierung des Isoniazids [8]. Pyrazinamid könnte auch einen Einfluss auf den Porphyrinstoffwechsel haben [11].

Die Therapie der Pellagra besteht in der Beseitigung der wahrscheinlichen Ursachen, wie z. B. dem Absetzen auslösender Medikamente und der Substitution von Vitamin B3 und Vitamin B6. Diese können auch prophylaktisch oder therapeutisch bei laufender Tuberkulostatika-Therapie gegeben werden [7]. Gleichwohl kann es auch hierunter gleichfalls zum Auftreten von Pellagra kommen $[1,2,5]$. Ein spezifische Lokaltherapie wird nicht empfohlen, lediglich unspezifisch pflegende Externa [9]. In einem Falle wurde aber über die erfolgreiche topische Anwendung von Nikotinamid im Bereich der Hautveränderungen berichtet [3]. Die Rolle von externen Lichtschutzmitteln wurde bislang noch nicht näher untersucht, jedoch spricht unser Fall mit promptem Ansprechen auf die Applikation eines Sunblockers dafür, dass diese wirksam sind und konsequent angewendet werden sollten.

Angesichts der wieder zunehmenden Bedeutung von Tuberkuloseerkrankungen [4] und der entsprechenden Zunahme von Patienten unter tuberkulostatischer Chemotherapie sollten Dermatologen sich der Möglichkeit von pellagroiden Erythemen hierunter bewusst sein und in Kooperation mit den behandelnden Pulmonologen frühzeitig intervenieren.

\section{Danksagung \\ $\nabla$}

Wir danken Herrn Dr. med. Michael Praus, Plauen, Labormedizinische Partnerschaft Dres. Scholz, Hummel, Praus, Grimmer und Schottmann für die Bestimmung des Vitamins B6 im Sammelurin.

\section{Abstract}

\section{Photosensitivity with Pellagroid During Tubercolosis Chemotherapy with Pyrazinamide $\nabla$}

We report on a 73-years old male caucasian patient who while being on antibiotic therapy due to tuberculosis developed a maculopapular drug induced exanthema and subsequently a pellagroid erythema in sunexposed areas. Pyrazinamide is supposed to be causal for this phenomenon. After six days of strict photoprotection and after three days of avoidance of the drug skin lesions resolved almost completely without further specific treatment. 


\section{Literatur}

1 Bender DA, Russel-Jones $R$. Isoniazid-induced pellagra despite vitamin-B6 supplementation. Lancet 1979; 2: 1125-1126

2 Burke GJ, Hlangabeza T. Isoniazid-induced pellagra in a patient on vitamin B supplement. S Afr Med J 1977; 14: 719

3 Comaish JS, Felix RH, McGrath H. Topically applied niacinamide in isoniazid induced pellagra. Arch Dermatol 1976; 112: 70 - 72

4 Crofts JP, Gelb D, Andrews N, Delpech V, Watson JM, Abubakar I. Investigating tuberculosis trends in England. Public Health 2008; Epub vor Druck

5 Darvay A, Basara T, McGregor JM, Russel-Jones R. Isoniazid induced pellagra despite pyridoxine supplementation. Clin Exp Dermatol 1999; 24: $167-169$

6 Hendricks WM. Pellagra and pellagra-like dermatoses: etiology, differential diagnosis, dermatopathology, and treatment. Semin Dermatol 1991; 10: $282-292$
7 Jørgensen J. Pellagra probably due to pyrazinamide: development during combined chemotherapy of tuberculosis. Int J Dermatol 1983; 22: $44-45$

8 Muratake T, Watanabe H, Hayashi S. Isoniazid-induced pellagra and the N-acetyltransferase gene genotype. Am J Psychiatry 1999; 156: 660

9 Pitche PT. Pellagre et érythèmes pellagroïdes. Santé 2005; 15: 205 208

10 Schmutz JL, Cuny JF, Trechot P, Weber M, Beurey J. Les érythèmes pellagroïdes médicamenteaux. Ann Dermatol Venereol 1987; 114: 569576

11 Treece GL, Magnusson CR, Patterson JR, Tschudy DP. Exacerbation of porphyria during treatment of pulmonary tuberculosis. Am Rev Respir Dis 1976; 113: $233-237$ 